Article

\title{
Predicting the Current and Future Suitable Habitat Distribution of Swimming Crab (Portunus trituberculatus) using the Species Distribution Model under Climate Change
}

\author{
Xingyu Liu, Xiaolu Han, Zhiqiang Han* \\ Fishery College, Zhejiang Ocean University, Zhoushan, Zhejiang, 316002, China; ccliuxingyu@163.com (X.L.); \\ 1084325960@qq.com(X.H.); hanzq@zjou.edu.cn (Z.H.) \\ * Correspondence: hanzq@zjou.edu.cn; Tel.: +86-580-2089333
}

\begin{abstract}
Simple Summary: Climate change has remarkably affected the marine ecosystem. The coastal area of China is particularly sensitive to climate change. Previous studies on the impacts of climate change on species' suitable habitats have ignored the seasonal changes of marine species distribution. In this study, we used the species distribution model to assess the seasonal distribution of Portunus trituberculatus under climate scenarios.
\end{abstract}

\begin{abstract}
Species have shown their habital variations in responding to climate change, especially during the spring and summer spawning seasons. The species distribution model (SDM) is considered the most favorable tool to study the potential effects of climate change on species distribution. Therefore, we developed the ensemble SDM to predict the changes in species distribution of Portunus trituberculatus among different seasons in 2050 and 2100 under the climate scenarios RCP4.5 and RCP8.5. The results of SDM indicate that the distribution of this species will move northward and have obviouse seasonal variations. Meanwhile, the suitable habitat for the species will be significantly reduced in summer, with loses rates ranging from $45.23 \%$ (RCP4.5) to $88.26 \%$ (RCP.8.5) by 2100 s. Habitat reduction will mainly occur in the East China Sea and southern part of the Yellow Sea, while there will be a small increase in the northern Bohai Sea. These findings will be important to manage the ecosystem and fishery, provide an information forecast of this species in the future, and maintain species diversity if the seawater temperature rises.
\end{abstract}

Keywords: Portunus trituberculatu, climate change, species distribution model, suitable habitat

\section{Introduction}

A large number of evidence show climate change can result in marine animals migrating northward to escape the heat, and loss of animal biomass. Shifts in the distribution of marine species from phytoplankton to marine mammals have been observed across all ocean regions [1]. For example, the distribution range of Gadus macrocephalus in the Yellow Sea has moved northward by $0.5^{\circ} \mathrm{N}$ since 1959 [2]. The alterations in the distribution of marine species likely resulted in community restructuring and ecosystem biodiversity losses [3]. It is projected to cause significant impacts on fisheries and human communities [4]. Besides the shift in distribution, the marine species life histories and life cycles have also been significant influenced by climate change [5]. Accordingly, it is important to predict spatial-tempora habitat distributions of marine species under future climate scenarios for exploring the potential impacts of climate change on coastal fisheries. This will improve our 
understanding of how marine species responding to changes in climatic conditions.

Swimming crab (Portunus trituberculatus), which belongs to phylum Crustacea, order Decapoda, and genus Portunus, is widely distributed in the coastal waters of China, Japan, and Korea. It is one of the most important commercial species in China, with a catch of 458,380 tons in 2019 [6]. This species has the typical habit of seasonal migration, and the distribution varies significantly among the seasons. Every spring, it comes to the shallow coastal waters of China (approximately 3-5 $\mathrm{m}$ deep) for spawning and growing. Then, in the autumn season, it gradually migrates to the deep waters (10-30 m deep) [7]. Previous studies have focused on the morphology, habits, reproduction, breeding, and migration of swimming crabs $[8,9,10]$.

Temperature and salinity are important abiotic factors affecting the growth, survival and molting of $P$. trituberculatus [11]. Liao et al. [12] showed that the optimum temperature and salinity of $P$. trituberculatus are $15{ }^{\circ} \mathrm{C}-30{ }^{\circ} \mathrm{C}$ and $15-35 \%$, respectively, and the high temperature exceeding $30{ }^{\circ} \mathrm{C}$ is fatal to this species. Dai et al. [13] pointed out that temperature increases could accelerate the molting of crabs and promote their development. But at the same time, energy expenditure and metabolism increase, and survival rate decreases. The distribution and biomass of $P$. trituberculatus may be closely related to climate factors. However, the potential impacts of climate change on the distribution of this species remain unevaluated. Therefore, it is necessary to study the impact of climate change on the distribution of $P$. trituberculatus, which can supply important data for developing future climate adaptation management strategies.

The species distribution model (SDM), which is based on the relationship between species occurrence and environmental variables, can describe and predict the distribution patterns of highly migratory marine species [14]. SDMs are widely recognized as important marine spatial planning tools. Xue et al. [15] used one SDM maximum entropy (MaxEnt) to predict the potential habitat distribution of Scomber japonicus in the Northwest Pacific Ocean during the peak fishing season (May to October) and revealed range shifts in different months. In addition, SDM can also anticipate possible effects of climate change scenarios on the distribution of marine species, given adequate predictions of key habitat variables [16].

The single SDM usually has greater uncertainty and non-universality [17,18]. The ensemble SDMs provided by the Biomod2 software [19] can sovle these shortcomings. It can map the main trend of the model (ie, average, median or other percentile) to overall change or uncertainty of all models. In this study, we developed an ensemble SDM integrating nine single SDM algorithms to predict the potential distribution of $P$. trituberculatus in the future by environmental data and biological data. We also tried to identify the major environmental variables determining species distribution.

\section{Materials and Methods}

\subsection{Swimming crab data}

P. trituberculatus occurrence data were obtained through four fishery resource surveys. From 1998 to 2000 , the "Beidou" fishery science survey vessel conducted four fishery resource surveys in the East China Sea, the Yellow Sea, and the Bohai Sea. In total, 306 occurrence records of swimming crab in spring were included, 296 records in summer, 275 records in autumn, and 290 records in winter [20].

\subsection{Environmental data}

Although the distribution and life cycle of marine species are influenced by many environmental 
factors, previous studies demonstrated a few variables could accurately predict species distribution $[21,23,24,25]$. Considering the biological relevance and data availability under current and future climate scenarios, five predictor variables sea surface temperature, surface salinity, current velocity, offshore distance, and depth were used in the SDM. Sea surface temperature, surface salinity, current velocity, offshore distance were extracted from the Bio-Ocean Rasters for Analysis of Climate and Environment (Bio-ORACLE, http://www.bio-oracle.org) $[25,26]$. Environmental variable depth was extracted from Global Marine Environment Dataset (http://gmed.auckland.ac.nz).

Temperature directly affected the physiological habit of swimming crab and varied greatly among seasons [27]. However, only mean annual sea surface temperature, the average temperature of the warmest month and coldest month are available in Bio-ORACLE. Therefore, the mean annual sea surface temperature represented the mean season surface temperature in spring and autumn, and the average temperature of the warmest month and coldest month were used for the environmental layers in summer and winter, respectively. We adopted the spatial resolution of $5 \times 5$ arcmin or higher and the geographic coverage of approximately $9.2 \times 9.2 \mathrm{~km}^{2}$ to match the five environmental data layers. The all pairwise Pearson correlation coefficient of the environmental data was below $|0.7|$ to remove prohibitive levels of redundancy among layers [16].

We retrieved the future temperature and salinity data layers under the typical concentration path emission scenarios (RCP) of RCP4.5 and RCP8.5 from Bio-ORACLE to predict the spatial distribution of $P$. trituberculatus in the coastal areas of China under climate change. Future layers were produced by averaging data from distinct AOGCMs provided by the CMIP5 [28]. This database provides two time series of years 2050 (2040-2050) and $2100(2090-2100)$ with the same spatial resolution of 5 arcmin for prediction environment variables [29]. The two-time series were representative of mid and long-term future climate conditions. RCP4.5 are intermediate emission scenarios, and RCP8.5 is a pessimistic scenario with higher concentrations. Therefore, two-time series (2050 and 2100) and two RCPs (RCP4.5 and RCP8.5) were considered to investigate potential future distributional shifts of swimming crab.

\subsection{Ensemble prediction model}

Nine models (GLM, generalized linear models; GBM, generalized boosted models; GAM, generalized additive models; CTA, classification tree analysis; ANN, artificial neural networks; SRE, surface range envelope; FDA, flexible discriminant analysis; RF, random forest; MaxEnt, maximum entropy) provided by Biomod2 software package were used to predict the suitable habitat of this species. Among ccurrence records, $80 \%$ of the data were used for modeling, and $20 \%$ were used for model validation. Each model was run three times, with a total of 27 times [30]. Kappa coefficient, true skill statistic (TSS), and receiver operating characteristic (ROC) were selected to evaluate the accuracy of the model [31]. We screen single models with TSS $>0.80$ and AUC $>0.9$ and use the weighted average method to construct a combined forecasting model. The weight of calculation results of single model was determined by its TSS value. The normalized results of a single model were multiplied by the corresponding weights, and the combined model was constructed to calculate the potential suitable habitat Index of the swimming crab in the study area.

The ensemble model predicted the probability (0-100\%) of the presence of $P$. trituberculatus in each grid at 5 arcmin resolution of the study area. To produce a presence/absence map of $P$. trituberculatus, continuous probability values were converted to binary predictions based on a 
threshold value of $30 \%$. The 'BIOMOD_RangeSize' function in Biomod2 package to investigate the potential shifts in suitable areas (i.e. suitable areas lost, remaining stable or gained) under RCP4.5 and RCP8.5 climate scenarios.

Pearson correlation analysis was applied to assess the relative importance of each variable in predicting species distribution [17]. Besides, to study the temperature sensitivity of species suitable habitat in summer, we simulated the trend of narrowing of species suitable habitat in summer under 0.2-2.0 ${ }^{\circ} \mathrm{C}$ warming, using $0.2{ }^{\circ} \mathrm{C}$ as a temperature increase step.

Table 1 The importance of environmental variables in the potentially suitable distribution of swimming crab.
Predictor variables
Contribution

$\begin{array}{ll}\text { Water depth } & 0.630 \\ \text { Temperature } & 0.326\end{array}$

Distance to shore

Salinity

0.067

Current velocity

0.026

\section{Results}

\subsection{Model assessment and environmental variable factor contribution}

The assessment of the developed model was conducted by TSS and ROC values. The TSS values for every single SDM varied from 0.73 (SRE) to 0.86 (GAM) (Figure 1). The ROC values ranged from 0.88 (SRE) to 0.94 (RF) (Figure 2). Considering the TSS and AUC values, GAM model gives the best predictive performance. Considering the cutoff values of TSS and ROC, all nine model algorithms were applied for further ensemble modeling. The TSS and AUC values of the ensemble model were $0.90 \pm 0.02$ and $0.97 \pm 0.2$, respectively. The predictive performance of the ensemble model exceeded the performance of all individual models, indicating a high level of accuracy in the ensemble model prediction. The results of the contribution of each environmental variable revealed that, among the five variables used for model development, depth $(>0.6)$ was the most contributor to the potential distribution of species, followed by temperature and offshore distance, salinity and current velocity $(<$ 0.1) contributed the least (Table 1). Response curves of temperature showed the suitable temperature range for this species varied greatly among seasons (Figure 3). The suitable temperature for years is between $5{ }^{\circ} \mathrm{C}$ and $28^{\circ} \mathrm{C}$. 


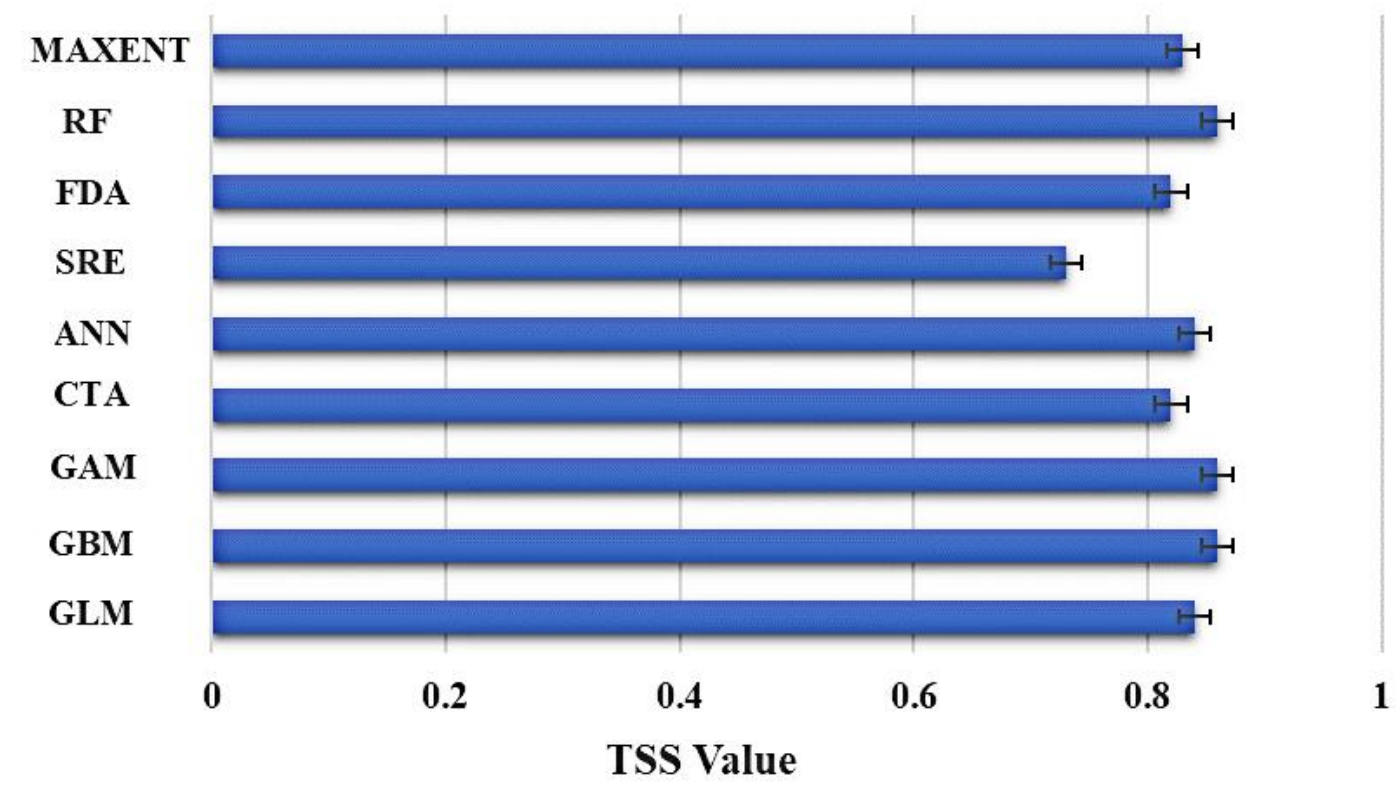

Figure 1 The true skill statistics (TSS) values for single models.

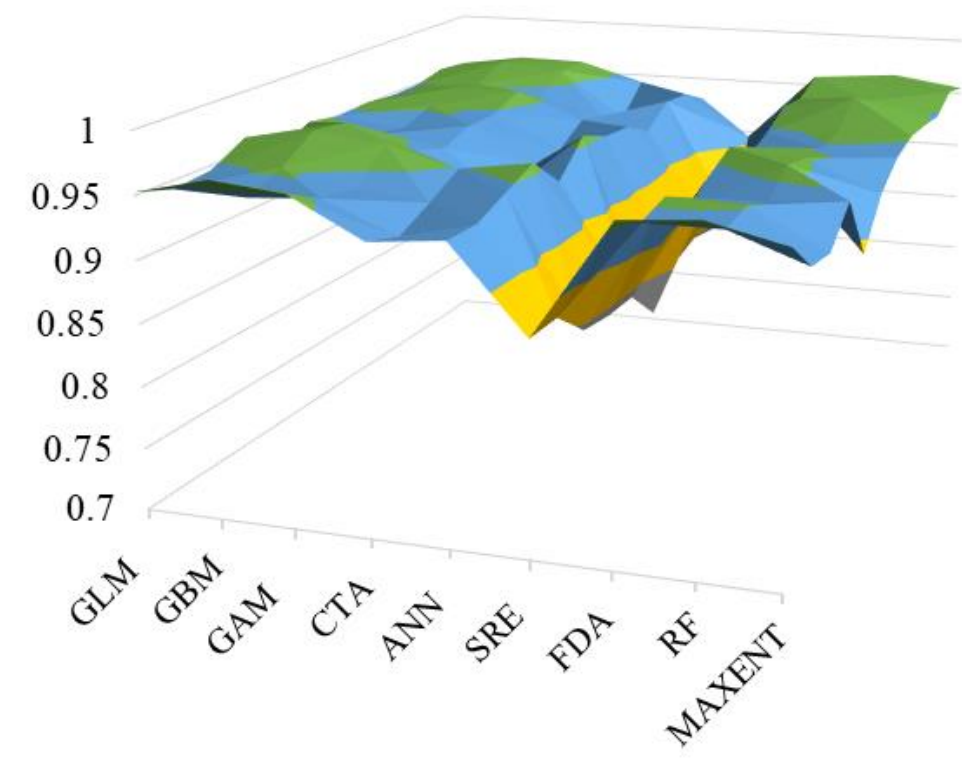

$\mathrm{AUC}=0.7-0.75=0.75-0.8=0.8-0.85=0.85-0.9=0.9-0.95 \approx 0.95-1$

Figure 2 Prediction accuracy of different models based on the ROC values. 

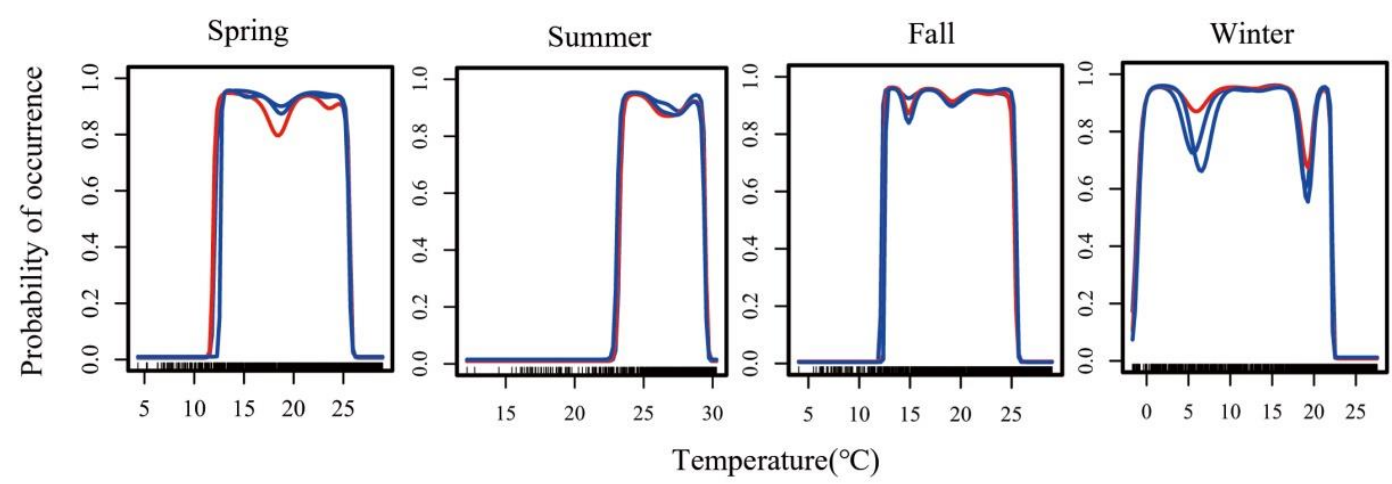

Figure 3 Response curves of predicted occurrence probability of Portunus trituberculatus against temperature based on the GAM model.

\subsection{Current and future potential distributions}

The predicted current suitable habitats of swimming crab under current climate conditions are shown in Figure 4. All the occurrence records were covered in the predicted current suitable habitat. Compared the four seasons of predicted suitable habitats of swimming crab, the Yellow Sea, and the East China Sea were suitable for this species with high habitat suitability in four seasons. The Bohai Sea showed low habitat suitability in the Fall season. Under future climate scenarios, the suitable habitat for swimming crab will decrease in spatial extent, especially in summer (Figure 5). Under RCP4.5 climate change scenario, a decrease of $4.47 \%$ (winter)-35.29\%(summer) were predicted in the suitable habitat in $2050 \mathrm{~s}$, while in $2100 \mathrm{~s}$, the loss of suitable habitat will be predicted from $3.37 \%$ (winter)to $45.23 \%$ (summer) (Table 2). The loss of potential distribution will be more aggravated under RCP8.5 scenario than RCP4.5. The loss of future potential distribution under RCP8.5 scenario also mainly happen in summer seasons, with $37.87 \%$ (2050s) to $88.25 \%$ (2100s). Under climate change, the loss of potential distribution mainly happens in the East China Sea (Figrue 5). The East China Sea is predicted to become less suitable for this species by 2100s.

Future predictions under RCP scenarios show that the suitable habitat of this species will shift northward. The changes in the distribution centroid of swimming crab are broadly similar under the RCP4.5 and RCP8.5 climate scenarios. The latitudinal centroid shifts significantly in summer than in other seasons under the climate scenarios (Table 3). The current latitudinal centroid of the potential habitat of swimming crab in summer is $31.92^{\circ} \mathrm{N}$. Under the RCP4.5 scenario, the centroid in summer will shift $2.31^{\circ}$ northward by the mid-21st century and then shift $0.86^{\circ}$ northward by the late $21 \mathrm{st}$ century. Under the RCP8.5 scenario, the north shift of centroid in summer is $2.49^{\circ}(2050 \mathrm{~s})$ and $13.31^{\circ}(2100 \mathrm{~s})$. Notably, the latitudinal centroid of the swimming crab habitat under the RCP8.5 scenario will be located to the north of its centroid under the RCP4.5 scenario. 
Table 2 Range variation of swimming crab in different seasons and periods based on ensemble SDM

\begin{tabular}{ccccc}
\hline Species range change & spring & summer & fall & winter \\
\hline RCP4.5-2050 & -5.52 & -35.29 & -5.60 & -4.47 \\
RCP4.5-2100 & -6.88 & -45.23 & -9.82 & -3.37 \\
RCP8.5-2050 & -2.81 & -37.87 & -2.18 & 2.83 \\
RCP8.5-2100 & -20.53 & -88.26 & -13.03 & -10.62 \\
\hline
\end{tabular}
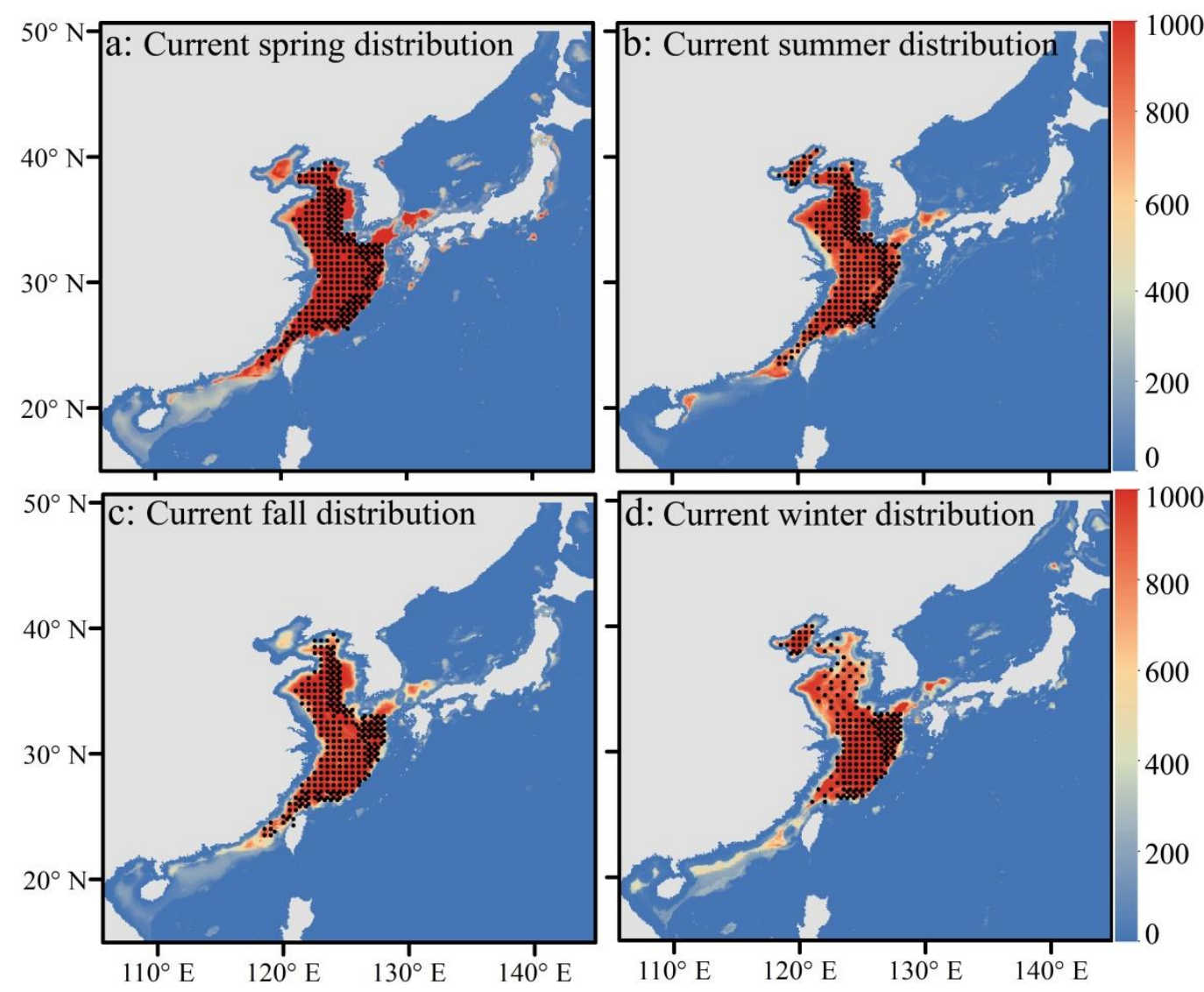

Figure 4 Predicted current potential distribution of Portunus trituberculatus. Black dots represent the occurrence records used to build the species distribution model. Color gradient indicates variation in habitat suitability (red $=$ highest, blue $=$ lowest).

We also investigated the temperature increase sensitivity of swimming crab in summer. It was found that the rate of habitat loss increased exponentially with increasing temperature. When the temperature rises by $0.2^{\circ} \mathrm{C}$ to $2^{\circ} \mathrm{C}$, the loss rate will rise from $2.8 \%$ to $80.5 \%$ (Figure 6). Notably, the loss rate drops sharply by $24 \%$ when the temperature rises by $1.2^{\circ} \mathrm{C}$, compared with the loss rate when 
the temperature rises by $1^{\circ} \mathrm{C}$.

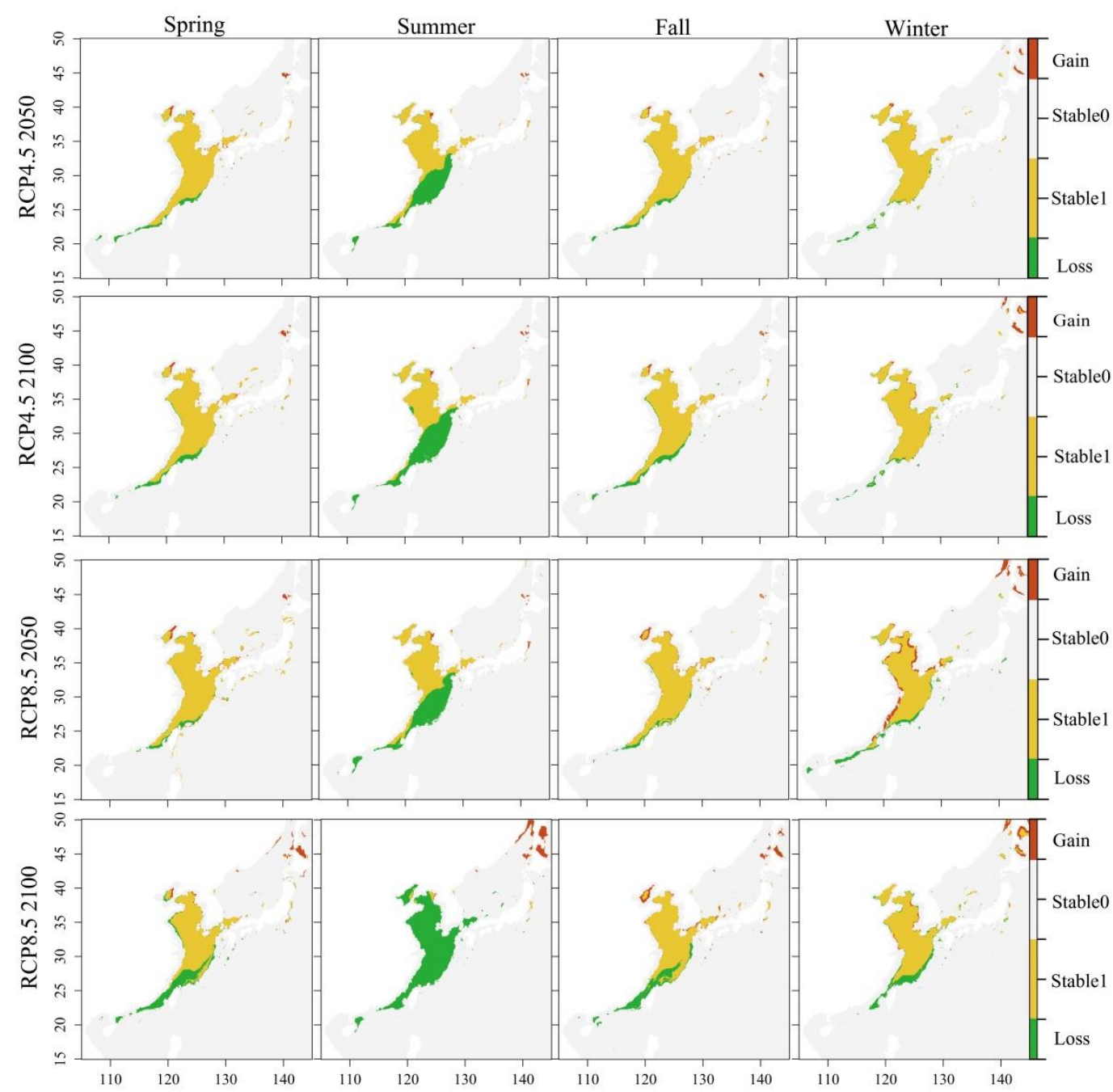

Figure 5 Changes of centroid distribution of swimming crabs in different seasons in 2050 and 2100 under RCP 4.5 and RCP 8.5.

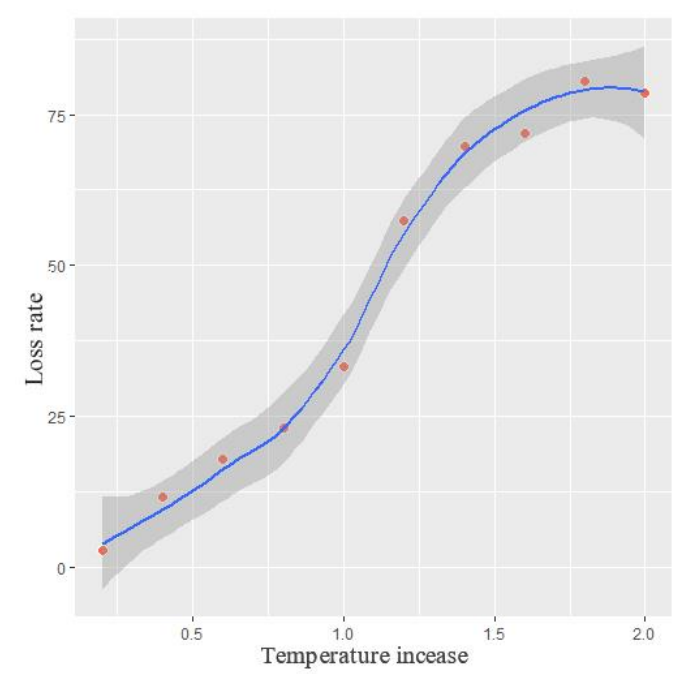

Figure 6 Habitat loss rate of swimming crabs in summer under temperature increase. 
Table 3 The latitudinal centroid of swimming crab under current and future climate conditions

\begin{tabular}{ccccc}
\hline & spring & summer & fall & winter \\
\hline Current & 31.71 & 31.92 & 31.54 & 32.39 \\
RCP4.5-2050 & 32.21 & 34.23 & 32.19 & 33.09 \\
RCP4.5-2100 & 32.66 & 35.09 & 32.51 & 33.52 \\
RCP8.5-2050 & 32.41 & 34.41 & 32.17 & 33.80 \\
RCP8.5-2100 & 33.93 & 45.23 & 32.17 & 34.67 \\
\hline
\end{tabular}

\section{Discussion}

\subsection{Prediction model accuracy and current distribution}

In this study, we first develped an ensemble SDM for swimming crab to predict seasonal potential suitable habitat of this species under current and future climate conditions. This may be the first study in the Northwestern Pacific, considering the seasonal distribution variation on the impact of climate change. Our ensemble SDM not only successfully predicted seasonally suitable habitat (i.e., survival probability) for $P$. trituberculatus, but also exhibits high performance. The AUC value, TSS, and kappa value of the ensemble model are more than $0.95,0.9$, and 0.8 , respectively.

Our ensemble SDM for $P$. trituberculatus showed excellent predictive performance and accurately predicted the current known distribution of this species. The present study indicates that the current potential habitat of $P$. trituberculatus is widely distributed across the Bohai Sea, the Yellow Sea, and the East China Sea, with the highest suitability found in the East China Sea(Figure 5). This result agreed well with fishing data [6]. East China Sea is major fishing ground for P. trituberculatus. Our ensemble predictions suggest that environmental conditions in the South China Sea are unsuitable for this species. Few reliable reports of this species in the South China Sea supported our predictions (Liao et al., 2008). No catch statistics about P. trituberculatus published in the South China Sea [6].

\subsection{Effects of climate change on suitable habitats}

The spatial variability of $P$. trituberculatus suitable habitat sustained previous empirical studies showing the effects of climate change on marine nekton distributional ranges and abundance $[32,33]$. The present model predicts a decrease in P. trituberculatus habitat suitability in the East China Sea, which indicates that climate change will result in a spatial redistribution of this species and a serious decline of the potential catches by 2050s and 2100s. However, the Bohai Sea will be more suitable than the Yellow Sea and the East China Sea for swimming crab under the RCP scenarios. Although both the Bohai Sea and the Yellow Sea are on the continental shelf, the warming seawater will compensate for the inherent lack of high latitude and low temperature in the Bohai Sea, attracting the population's migration. The predicted shift of range is biologically plausible under climate change. According to our results, the high water temperature will force the northward distribution of swimming crab in the East China Sea. Water temperature can affect various physiological processes of swimming crab in growth, survival and molting. High water temperature exceeding $30^{\circ} \mathrm{C}$ can lead to larval development arrest of P. trituberculatus [12]. This thermal tolerance limit would certainly influence the geographical distribution of this species. In addition, with the severe loss of suitable habitat for swimming crab, its migratory route will be shortened. Even their migratory habits will disappear, thus changing their life 
history.

The shift of the centroid of the summer suitable habitat to the north may be one of its reproductive strategies to cope with climate change, but there will also be inevitable losses. Simply changing the timing and location of spawning may result in a mismatch between feeding grounds and areas of high phytoplankton density. Moreover, it is difficult to predict whether new spawns can provide necessary sediments and corresponding hydrological characteristics for egg deposition [34]. Therefore, the study on the change of suitable habitat in summer needs to be further deepened.

\subsection{Implication for fishery management}

Future P. trituberculatus habitat distribution predictions for the 2050-2100 period based on RCP climate change scenarios became less favourable due to the negative effects of warming. The distribution range shifts and loss of habitat distribution will reduce species fishery catche of $P$. trituberculatus and ultimately influence the local fisheries activities. Further studies are required to investigate the effects of climate change on fisheries catches in the East China Sea. Conservation and management actions must be required to mitigate the negative consequences of the predicted climate change scenario on $P$. trituberculatus in the coastal areas of China. The socioecological system must consider adaptation actions to address the impacts of future climate scenarios along the China coast and other parts of the world.

Author Contributions: Conceptualization, Z.H.; Methodology, Z.H.; Validation, Z.H.; Formal analysis, X. Y. and X.H.; Resources, Z.H.; Writing — original draft preparation, X.Y. and X.H.; Writing—review and editing, X.Y.; Supervision, Z.H.; Project administration, Z.H.; Funding acquisition, Z.H.

Funding: This research was funded by the National Key Research and Development Program of China (2017YFA0604902), and Zhejiang Provincial Natural Science Foundation of China (LR21D060003).

Conflicts of Interest: The authors declare no conflict of interest.

Table 2 Range variation of swimming crab in different seasons and periods based on ensemble SDM

Table 3 The latitudinal centroid of swimming crab under current and future climate conditions

\section{References}

1. Poloczanska, E.S. et al., 2016: Responses of Marine Organisms to Climate Change across Oceans. Front. Mar. Sci., 3(28), 515, doi:10.3389/fmars.2016.00062.

2. Li, Z.L.; Jin, X.S.; Zhang, B.; Zhou, Z.P.; Shan, X.J.; Dai, F.Q. Interannual variation of population characteristics of Gadus macrocephalus in the Huanghai Sea [J]. Oceanologia et limnologia sinica, 2012,43 (05): 924-931 (in Chinese)

3. Rilov, G., 2016: Multi-species collapses at the warm edge of a warming sea. Sci. Rep., 6, 36897 , doi:10.1038/srep36897

4. Claudio Silva, Francisco Leiva, José Lastra. Predicting the current and future suitable habitat distributions of the anchovy (Engraulis ringens) using the Maxent model in the coastal areas off central-northern Chile[J]. Fisheries Oceanography, 2019, 28.

5. Merino, G., Barange, M., Blanchard, J. L., Harle, J., Holmes, R., Allen, I., ... Rodwell, L. D. (2012). Can marine fisheries and aquaculture meet fish demand from a growing human population in a changing climate? Global Environmental Change, 22(4), 795-806. https://doi.org/10. 1016/j.gloenvcha.2012.03.003

6. Fisheries Bureau of Ministry of Agriculture (2020). China fishery statistical yearbook (p. 43). Beijing, China: 
China Agriculture Press.

7. Dai, A.Y.; Feng Z.G.; Song Y,Z. et al. Preliminary investigation of fishery biology of Portunus trituberculatus [J]. (in Chinese) Journal of Zoology, 1977 (02): 30-33. https://doi.org /10.13859/j.cjz.1977.02.015

8. Lu, S.; Li, R.; Zheng, W.; Chen, L.; Ren, Z.; Mu, C.; Wang, C. (2019). Long-term low-salinity stress affects growth, survival and osmotic related gamma-aminobutyric acid regulation in the swimming crab Portunus trituberculatus. Aquaculture Research. https://doi.org/10.1111/are.14242

9. Su, X.P.; Liu, J.J.; Wang, F.; Wang, Q.H.; Zhang, D.; Zhu, B .S.; Liu, D.P. Effect of temperature on agonistic behavior and energy metabolism of the swimming crab (Portunus trituberculatus), Aquaculture, 10.1016/j.aquaculture.2019.734573, (734573), (2019). https://doi.org/10.1111/are.12239

10. Fu, S.L.; Ding, M.M.; Liang, Q.J.; Yang, Y.J.; Chen, M.; Wei, X.F. Anli Wang, Shaoan Liao and Jianmin Ye, The key differentially expressed genes and proteins related to immune response in the spleen of pufferfish (Takifugu obscurus) infected by Aeromonas hydrophila, Fish \& Shellfish Immunology, 10.1016/j.fsi.2019.05.016, (2019). （in chinese） https://doi.org/10.1111/are.13155

11. Shaokun $\mathrm{Lu}$ Ronghua Li Weibing Zheng Lian Chen Zhiming Ren Changkao Mu Weiwei Song Chunlin Wang, Long-term low-salinity stress affects growth, survival and osmotic related gammaaminobutyric acid regulation in the swimming crab Portunus trituberculatus, 2019

12. Liao, Y.Y.; Xu, Z. Y.; Wu, B.D. Effects of salinity and temperature on survival and feeding of Portunus trituberculatus [J]. Journal of Ecology, 2010, 30 (13): 3396-3405. (in chinese)

13. Dai, C.; Wang, F; Fang ZH. Effect of temperature on respiratory metabolism and enzyme activity in Portunus trituberculatus. Progress in Fishery Sciences 2(2014):90-96. (in Chinese)

14. Elizabeth A. Becker, James V. Carretta Karin A. Forney Jay Barlow Stephanie Brodie Ryan Hoopes Michael G. Jacox Sara M. Maxwell Jessica V. Redfern Nicholas B. Sisson Heather Welch Elliott L. Hazen Performance evaluation of cetacean species distribution models developed using generalized additive models and boosted regression trees[J]. Ecology and Evolution, 2020.

15. Xue, J.L.; Fan, w.; Tang, F.H et, al. Analysis of potential habitat distribution of Scomber japonicus in northwest Pacific Ocean using maximum entropy model [J]. South China Fisheries Science, 2018, (1):92-98 (in Chinese). https://doi.org /10.3969/j.issn.2095 - 0780.2018.01.012

16. Zhang Zhixin, Shengyong Xu, César Capinhac, Robbie Weteringsd, Tianxiang Gaob, Using species distribution model to predict the impact of climate change on the potential distribution of Japanese whiting Sillago japonica , 2019

17. Zhang,L.; Liu, Shi.; Sun, P.s. et al. Segmentation and mapping of uncertain components in the simulation of the impact of climate change on species distribution: a case study of Pinus tabulaeformis [J]. Acta Ecologica Sinica, 2011, 31 (19): 5749 - 5761(in chinese)

18. Wilfried T. BIOMOD-optimizing predictions of species distributions and projecting potential future shifts under global change[J]. Global Change Biology, 2003(10): 1353-1362.

19. Thuiller, W., Georges, D., Engler, R., 2014. biomod2: ensemble platform for species distribution modeling. R package version 3.3.7.

20. Jin, X .S.; Cheng, J. S.; Qiu, S. Y. et al. Integrated research and evaluation on fisheries resources in Huanghai Sea and Bohai Sea[M]. Beijing: Ocean Press, 2006: 350-357 (in Chinese).

21. Goldsmit, J.; Archambault, P.; Chust, G.; Villarino, E.; Liu, G.; Lukovich, J.V.; Barber, D.V. Howland, K.L. Projecting present and future habitat suitability of shipmediated aquatic invasive species in the Canadian Arctic. Biol. Invasions. 2018, 20, 501-517. https://doi.org/10.1007/s10530-017-1553-7 
22. Bosch, S.; Tyberghein, L.; Deneudt, K.; Hernandez, F.; De Clerck, O. In search of relevant predictors for marine species distribution modelling using the MarineSPEED benchmark dataset. Divers. Distrib. 2018, 24, 144-157. https://doi.org/10.1111/ddi.12668

23. Belanger, C.L.; Jablonski, D.; Roy, K.; Berke, S.K.; Krug, A.Z.; Valentine, J.W. Global environmental predictors of benthic marine biogeographic structure. Proc. Natl. Acad. Sci. 2012, 109, 14046-14051. https://doi.org /10.1073/pnas.1212381109

24. Assis, J.; Tyberghein, L.; Bosch, S.; Verbruggen, H.; Serrão, E.A.; De Clerck, O. Bio-ORACLE v2. 0: extending marine data layers for bioclimatic modelling. Glob. Ecol. Biogeogr. , 2018,27, 277-284. ._ https://doi.org /10.1111/geb.12693

25. Basher, Z.; Bowden, D.A.; Costello, M.J. 2014. Global marine environment dataset (GMED). Version 1.0 (Rev.01.2014). http://gmed.auckland.ac.nz (accessed 30 October 2018)

26. Morley, J. J.; Heusser, L. E. Role of orbital forcing in east Asian monsoon climates during the last 350 kyr: Evidence from terrestrial and marine climate proxies from core RC14-99, Paleoeanography, 1997, 12(3),483-493, https://doi.org /10.1029/97PA00213.

27. Doney, S.C.; Ruckelshaus, M.; Duffy, J.E.; Barry, J.P.; Chan, F.; English, C.A.; Galindo, H.M.; Grebmeier, J.M.; Hollowed, A.B.; Knowlton, N.; Polovina, J.; Rabalais, N.N.; Sydeman, W.J.; Talley, L.D. Climate change impacts on marine ecosystems. Ann. Rev. Mar. Sci. 2012, 4, 11-37. https://doi.org/10.1146/annurev-marine-041911-111611

28. Assis, J.; Tyberghein, L.; Bosh, S.; Verbruggen, H.; Serrão, E. A.; De Clerck, O. Bio-ORACLE v2.0: Extending marine data layers for bioclimatic modelling. Global Ecology and Biogeography. 2017. https://doi.org/10.1111/geb.12693

29. Tyberghein, L.; Verbruggen, H.; Pauly, K.; Troupin, C.; Mineur, F. De Clerck O Bio-ORACLE: A global environmental dataset for marine species distribution modelling. Global Ecology and Biogeography,2012, 21, 272-281. https://doi.org/10.1111/j.1466-8238.2011.00656.x

30. Singh K; Mcclean, C. J.; Büker, P. et al. Mapping regional risks from climate change for rainfed rice cultivation in India[J]. Agricultural Systems, 2017, 156: 76-84.

31. Lobo, J. M.; Jiménez,Valverde A; Real, R. AUC:a misleading measure of the performance of predictive distribution models. Global Ecology and Biogeography,2008,17(2),

145-151. https://doi.org/10.1111/j.1466-8238.2007.00358.x

32. Cheung, W. W. L., Lam, V. W. Y., Sarmiento, J. L., Kearney, K., Watson, R., Zeller, D., \& Pauly, D. (2010). Large-scale redistribution of maximum fisheries catch potential in the global ocean under climate change. Global Change Biology, 16, 24-35. https://doi.org/10.1111/(ISSN)1365-2486

33. Yáñez, E., Hormazabal, S., Silva, C., Montecinos, A., Barbieri, M. A., Valdenegro, A., ... Gómez, F. A. (2008). Coupling between the environment and the pelagic resources exploited off North Chile: Ecosystem indicators and a conceptual model. Latin American Journal of Aquatic Research, 36(2), 159-181

34. Wootton, Carl Smith. Reproductive Biology of Teleost Fishes (Wootton/Reproductive Biology of Teleost Fishes) || Gametogenesis. Reproductive Biology of Teleost Fishes. 2014, 10.1002/97. https://doi.org/ $\underline{10.1002 / 9781118891}$ 